\title{
Comparison of the efficacy of lotrafilcon $B$ and comfilcon A silicone hydrogel bandage contact lenses after transepithelial photorefractive keratectomy
}

\author{
Nancy M. Lotfy ${ }^{1}$, Tariq Alasbali ${ }^{2,3}$, Abdulrahman M. Alsharif ${ }^{2}$, Saeed M Al-Gehedan ${ }^{2}$, Sabah Jastaneiah ${ }^{2}$,
} Ashraf Al-Hazaimeh ${ }^{2}$, Hiba Ali ${ }^{2}$ and Rajiv Khandekar ${ }^{2,4}$

${ }^{I}$ Department of Ophthalmology, Faculty of Medicine, Cairo University, Egypt

${ }^{2}$ Department of Ophthalmology, Specialised Medical Centre Hospital, Riyadh, Saudi Arabia

${ }^{3}$ Al-Imam Muhammad Ibn Saud Islamic University College of Medicine, Riyadh, Saudi Arabia

${ }^{4}$ Research Department, King Khaled Eye Specialist Hospital, Riyadh, Saudi Arabia

\begin{abstract}
Background: At completion of transepithelial photorefractive keratectomy ( $t-P R K)$ surgery, the eye is usually fitted with a bandage contact lens to reduce discomfort and promote epithelial healing. This study aimed to compare the outcomes of eyes fitted with lotrafilcon B versus comfilcon A, silicone hydrogel bandage contact lenses after t-PRK for the correction of low to moderate myopia, with or without astigmatism.

Methods: In this comparative, prospective study, patients with myopia < -6 D with or without astigmatism (<1.75 D), who underwent t-PRK between January and June 2018, were randomly allocated to the lotrafilcon $\mathrm{B}$ and comfilcon A groups. Preoperative characteristics, including age, sex, eye treated, uncorrected visual acuity (UCVA), best-corrected visual acuity, mesopic pupil size, central corneal thickness, and refractive error were recorded. Postoperatively, pain score, UCVA, and corneal epithelial defect size on days 1, 4, and 7 were compared between the two groups.

Results: Twenty-nine eyes were included in each group. Demographic characteristics and preoperative measurements were similar between the two groups. UCVA was significantly improved on day 7 as compared to day 1 in the comfilcon A group $(P=0.03)$, but remained the same in the lotrafilcon B group $(P=0.70)$ as on day 1 postoperatively. There was no significant difference in UCVA between the two groups at any follow-up visits (all $P>0.05$ ). The pain score on the first postoperative day was significantly higher in the lotrafilcon B-fitted eyes than in the comfilcon A group $(P<0.001)$, but was significantly reduced in both groups compared to day 1 (both $P<0.001$ ). The epithelial defect in the comfilcon A group was significantly greater than in the lotrafilcon B group $(P<0.001)$ at day 1 postoperatively, with significant improvement in both groups $($ both $P<0.001)$.

Conclusions: Healing responses were better with lotrafilcon $B$ than with comfilcon A bandage contact lenses. The patients had a greater mean pain score with lotrafilcon B than with comfilcon A lenses on the first postoperative day, yet the final outcome was comparable between the two groups. We did not encounter any postoperative complications related to contact lens wear.

KEYWORDS

silicone hydrogel, bandage contact lenses, lotrafilcon B, comfilcon A, cornea, epithelial defect, pain score, transepithelial photorefractive keratectomy, t-PRK, PRK, prospective study
\end{abstract}

Correspondence: Nancy M. Lotfy, MD, Department of Ophthalmology, Faculty of Medicine, Cairo University. Al-Saray Street, El Manial, Postal Code: 11956, Cairo, Egypt. Email: nancymaher@kasralainy.edu.eg. ORCHID iD: https://orcid.org/0000-0002-9159-0020

How to cite this article: Lotfy NM, Alasbali T, Alsharif AM, Al-Gehedan SM, Jastaneiah S, Al-Hazaimeh A, Ali H, Khandekar R. Comparison of the efficacy of lotrafilcon B and comfilcon A silicone hydrogel bandage contact lenses after transepithelial photorefractive keratectomy. Med Hypothesis Discov Innov Ophthalmol. 2021 Summer; 10(2): 43-49. https://doi.org/10.51329/mehdiophthal1420

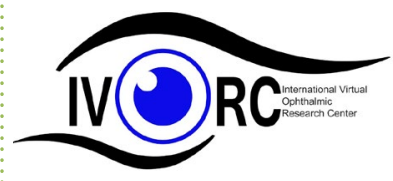

Copyright (C) Author(s). This is an open-access article distributed under the terms of the Creative Commons Attribution-NonCommercial 4.0 International License (http://creativecommons.org/licenses/by-nc/4.0/) which permits copy and redistribute the material just in noncommercial usages, provided the original work is properly cited. (c) (i) (s) 


\section{INTRODUCTION}

Photorefractive keratectomy (PRK) is a flapless technique used to treat refractive errors in patients with thin corneas, epithelial basement membrane disease, enlarged pupils, topographic irregularities, and patients who are not good candidates for laser-assisted in situ keratomileusis (LASIK) surgery [1].

There are various methods for epithelial removal prior to PRK, including mechanical debridement, laser removal (transepithelial photoablation), chemical de-epithelialization using a dilute alcohol solution, or a combination of these techniques [2-5]. The disadvantages of the procedure include prolonged visual recovery, pain, irregular epithelial healing, and corneal haze. Adequate management of these problems not only improves patient comfort, but also prevents the development of complications, such as corneal infections and haze [6]. Many modifications have been introduced to decrease these complications, and preservation of the epithelium is the mainstay of these techniques [7].

Transepithelial PRK ( $t$-PRK) was introduced as an alternative to conventional PRK. It is associated with less pain, faster recovery, and less haze than mechanical debridement or alcohol-assisted removal [7]. At completion of surgery, the eye is usually fitted with a bandage contact lens to reduce discomfort and promote epithelial healing. Often, silicone bandage contact lenses are used after PRK due to their higher oxygen permeability as compared to conventional hydrogel lenses, which allows extended wear. They provide faster re-epithelialization, decreasing the postoperative risk of infections, reducing patient discomfort and pain, and allowing earlier visual rehabilitation [1].

The use of postoperative bandage contact lenses is advised for 3-5 days because the estimated time of epithelial healing is approximately 2-4 days, and the time for resolution of pain and discomfort is about 1-3 days [8]. Silicone hydrogel lenses have extensively replaced conventional hydrogel lenses, because of their higher DK/t or oxygen transmissibility. The FDA-approved lenses that are used after PRK include lotrafilcon A, comfilcon A, lotrafilcon B, balafilcon A, senofilcon A, omafilcon A, and samfilcon A. Nevertheless, there are no clear conclusions regarding the best lens for use after PRK, in terms of better comfort and faster healing. Several studies have compared the healing times and comfort of different bandage contact lenses $[1,9,10]$.

However, to the best of our knowledge, no study has evaluated an Arab cohort of patients who face unique dry and dusty environmental conditions in countries such as Saudi Arabia. We thus compared the clinical performance of lotrafilcon B and comfilcon A silicone hydrogel bandage contact lenses after t-PRK for the correction of low to moderate myopia, with or without astigmatism, in a cohort of patients in Saudi Arabia.

\section{METHODS}

This was a prospective, contralateral eye comparison of two types of silicone hydrogel bandage contact lenses after t-PRK for the correction of low to moderate myopia, with or without astigmatism. The study was performed at the Ophthalmology Department of a private hospital (the Specialised Medical Center) in Saudi Arabia, from January to June 2018. All patients provided written informed consent prior to recruitment. This study was approved by the Research Ethics Committee of the Specialised Medical Center. Patients were included if they had myopia $<-6.00$ diopters (D), with or without astigmatism $(<1.75 \mathrm{D})$, with no ocular disease or amblyopia, no systemic diseases, no history of medications that might affect epithelial healing or perception of pain, and no history of ocular trauma or ophthalmic surgery.

Based on a previous study we estimated that the mean \pm standard deviation $(\mathrm{SD})$ of the pain score would be $2.25 \pm 0.96$ in eyes with lotrafilcon B and $1.64 \pm 067$ in eyes with comfilcon A bandage contact lenses [1]. The StatCalc of OpenEpi software (Centers for Disease Control and Prevention, Atlanta, GA, USA) was used to calculate the sample size. Twenty-nine eyes of 29 patients were required in each group to achieve an appropriate $95 \%$ confidence interval (CI) and $80 \%$ power. Primary outcome measures included postoperative uncorrected visual acuity (UCVA), pain score, and healing of corneal epithelial defects. Secondary outcome measures included any contact lens-related complications.

Preoperatively, data were collected on patient demographics, UCVA, best-corrected visual acuity (BCVA), cycloplegic refraction, corneal topography (Pentacam HR; Oculus Optikgeräte GmbH, Wetzlar, Germany), slitlamp biomicroscopy (Topcon, Livermore, CA, USA), and mesopic pupil diameter (Sirius, Schwind eye-techsolutions $\mathrm{GmbH}$ and Co.KG, Kleinostheim, Germany), Goldmann tonometry, and dilated fundoscopy.

All surgeries were performed by a single surgeon (T.A.) under sterile conditions and under topical anesthesia using oxybuprocaine preservative-free eye drops. A single-step t-PRK, in which the epithelium and stroma are ablated in a single step, was performed using a Schwind Amaris 500E excimer laser (Schwind eye-tech-solutions, Kleinostheim, Germany), followed by the application of $0.02 \%$ mitomycin C (MMC) for up to $15 \mathrm{~s}$, and copious 
irrigation with cold balanced salt solution. At the end of the procedure, one eye was randomly (decided by flipping a coin) selected to be fitted with the lotrafilcon B (Air Optix; Ciba Vision, Duluth, GA, USA) bandage contact lens (base curve $8.6 \mathrm{~mm}$, diameter $14.2 \mathrm{~mm}$, power $0.50 \mathrm{D}$ ), while the comfilcon A (Biofinity; CooperVision, San Ramon, CA, USA) bandage contact lens (base curve $8.6 \mathrm{~mm}$, diameter $14.0 \mathrm{~mm}$, power $0.50 \mathrm{D}$ ) was placed on the fellow eye. The characteristics of the bandage contact lenses are presented in Table 1. Patients, surgeons, and examiners were blinded to the randomized choice of the fitted bandage contact lens.

Postoperatively, both eyes received $1 \%$ prednisolone acetate eye drops (Pred Forte, Allergan, Irvine, CA, USA) four times/day for 4 weeks, gatifloxacin $0.3 \%$ eye drops (Zymar, Allergan, Inc., Irvine, CA, USA) four times/day for 5 days, ketorolac trometamol 0.5\% eye drops (Brown \& Burk UK Ltd., Hounslow, UK) four times/day for 5 days, and Systane ${ }^{\circledast}$ lubricant eye drops (Alcon Laboratories, Fort Worth, TX, USA) for 1 week. All patients were advised to take 1-2 tablets of oral ibuprofen $400 \mathrm{mg}$, if required, for pain control.

Postoperative assessment of UCVA, pain, and epithelial defects was scheduled on days 1, 4, and 7. The same physician performed preoperative and postoperative assessments. At each follow-up visit, UCVA was measured, using the Snellen chart with decimal notation, and slit-lamp bio-microscopy examination was performed. Pain perception was measured using a 5-point numeric rating scale, with 0 indicating "no pain" and 4 indicating "very severe pain" [12]. Corneal epithelial defects were measured using a slit-lamp scale (in $\mathrm{mm}$ ), with a narrow slit beam in the vertical and horizontal directions [13].

The data were collected on a pretested form and were transferred to an Excel" spreadsheet (Microsoft Corp., Redmond, WA, USA). Statistical analyses were performed using the Statistical Package for Social Studies (Version 22.0, SPSS; IBM Corp., Armonk, NY, USA). For quantitative variables, if the distribution was normal, we estimated the mean and SD, and for comparison, we calculated the two-sided $P$-value. Matched-pair analysis was used to compare the outcomes of the two types of bandage contact lenses. For qualitative variables, a two-sided $P$-value, was calculated from $2 \times 2$ tables using OpenEpi software. Chi-square values and two-sided $P$-values were used for evaluating more than two independent variables. UCVA, pain score, and the size of the epithelial defect at different follow-up visits were compared between groups and within each group. Statistical significance was set at $P<0.05$.

\section{RESULTS}

Fifty-eight eyes of 29 patients were included in this study. Figure 1 illustrates the patients' allocation to the lotrafilcon B or comfilcon A silicone hydrogel bandage contact lens group following t-PRK. Patient demographics and preoperative measurements in both groups are shown in Table 2. Preoperative parameters were similar between the groups. The mean \pm SD of the duration of $t-P R K$ for the comfilcon A and lotrafilcon $B$ groups was $53.6 \pm 6$ seconds and $51.3 \pm 20.8$ seconds, respectively $(P=0.20)$. The ablation zone in both eyes was $8.00 \pm 0.60 \mathrm{~mm}$.

Table 3 presents the intra- and inter-group comparisons of UCVA, epithelial defect size, and pain score postoperatively. UCVA had improved significantly by day 7 as compared to day 1 in the comfilcon A group ( $P$ $=0.03$ ), but no significant change was noted at day 7 as compared to day 1 postoperatively in the lotrafilcon $\mathrm{B}$ group $(P=0.70)$. There was no significant difference in UCVA between the two groups at all follow-up visits (all $P>0.05$ ). Epithelial defects in eyes fitted with comfilcon A were significantly greater than in eyes fitted with lotrafilcon $\mathrm{B}$ lenses $(P<0.001)$ on postoperative day 1 , but a significant improvement in both groups was noted by the end of the observation period (both $P<0.001$ ). The pain score in the lotrafilcon $B$ group was significantly higher on postoperative day 1 than on comfilcon $\mathrm{A}(P<0.001)$, but was comparable on day 4 and day 7 postoperatively (both $P>0.05$ ). No contact lens-related postoperative complications were noted in either study group at any follow-up visit.

Table 1. Characteristics of Silicone hydrogel bandage contact lenses [6] used following transepithelial photorefractive keratectomy (t-PRK) in present study

\begin{tabular}{|l|l|l|}
\hline Material & comfilcon A & lotrafilcon B \\
\hline Proprietary name & Biofinity & O2 Optix/Air Optix \\
\hline Manufacturer & CooperVision & CIBA Vision \\
\hline Surface treatment & None & Plasma coating \\
\hline Water content & $48 \%$ & $33 \%$ \\
\hline Dk & 128 & 110 \\
\hline Dk/t & 160 & 138 \\
\hline Modulus (Mpa) & 0.8 & 1.0 \\
\hline
\end{tabular}

Abbreviations: DK: D, material diffusivity; K, material solubility; DK/t [11], oxygen transmissibility, which is permeability (Dk) divided by lens thickness $(\mathbf{t})$. 


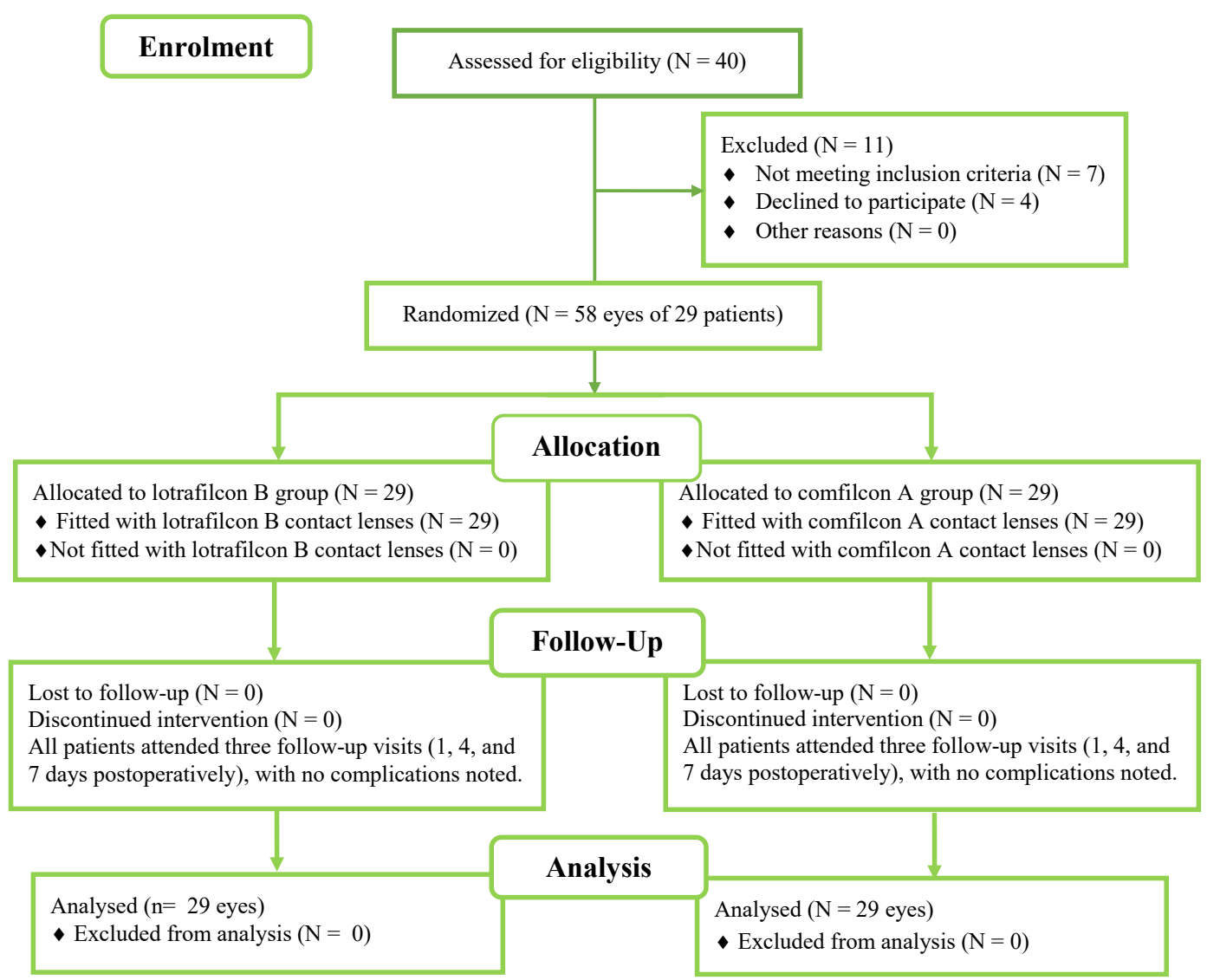

Figure 1. Patient allocation to the lotrafilcon B or comfilcon A silicone hydrogel bandage contact lens group following transepithelial photorefractive keratectomy (t-PRK) for the correction of myopia, with or without astigmatism.

\section{DISCUSSION}

No previous study has reported a comparison of two types of silicone hydrogel bandage contact lenses inserted following t-PRK for low to moderate myopia in Saudi patients. Patients who received lotrafilcon B lenses experienced more pain on postoperative day 1 than did patients who received the comfilcon A lens. However, the epithelial defect was greater in eyes with comfilcon A lenses than in those with lotrafilcon B lenses on day 1. Over time, all indicators suggested normal recovery and good postoperative outcomes in eyes with either type of bandage contact lens. We did not encounter any postoperative complications related to contact lens wear.

The Saudi population is exposed to unique risk factors that result in an unusually high prevalence of dry eye disease [14]. Hence, performing refractive surgery in this patient population is a challenge in itself. Therefore, adequate postoperative corneal oxygenation is fundamental for uneventful recovery in this particular population. Silicone bandage contact lenses with higher $\mathrm{DK} / \mathrm{t}$ values are beneficial in these environments [15]. A number of companies market bandage contact lenses with claims of product superiority. The present study confirmed the findings of widely varying patient discomfort and corneal healing, despite similar treatments and bandage contact lenses in the same patient.

The pain score was significantly greater in the lotrafilcon B group than in the comfilcon A group in our study at early follow-up. Eliacik et al. [1] also reported higher patient discomfort with lotrafilcon B lenses than with comfilcon A lenses after PRK. This may be due to the higher $\mathrm{DK} / \mathrm{t}$ value for comfilcon A lenses compared to lotrafilcon B lenses [6]. Moreover, lens deposits are a plausible factor in increased contact lens discomfort, which varies based on the lens material. In an in vitro study, comfilcon A had a uniform lipid deposition profile on day 1 of incubation in an artificial tear solution; however, lotrafilcon B revealed more lipid deposition on the surface than in the core [16]. These two differences between comfilcon A and lotrafilcon B lenses may explain the significantly higher pain score on postoperative day 1 in eyes fitted with the latter lens. 
Table 2. Comparison of preoperative characteristics of eyes treated with transepithelial photorefractive keratectomy, followed by fitting with two types of bandage contact lenses

\begin{tabular}{|c|c|c|c|c|}
\hline \multirow{3}{*}{$\begin{array}{l}\text { Variable } \\
\text { Sex, n (\%) }\end{array}$} & \multicolumn{2}{|r|}{$\begin{array}{c}\text { Comfilcon A } \\
\quad(\mathbf{n}=29)\end{array}$} & $\begin{array}{c}\text { Lotrafilcon B } \\
\quad(\mathbf{n}=29)\end{array}$ & \multirow{2}{*}{$\begin{array}{l}\boldsymbol{P} \text {-value } \\
0.990\end{array}$} \\
\hline & Male & $12(41.4)$ & $12(41.4)$ & \\
\hline & Female & $17(58.6)$ & $17(58.6)$ & \\
\hline \multirow[t]{2}{*}{ Eye, n (\%) } & Right & $15(51.7)$ & $14(48.3)$ & \multirow[t]{2}{*}{0.800} \\
\hline & Left & $14(48.3)$ & $15(51.7)$ & \\
\hline \multirow[t]{9}{*}{ Preoperative UCVA in decimal, $\mathbf{n}(\%)$} & 0 & $1(3.4)$ & $0(0.0)$ & \multirow[t]{9}{*}{0.600} \\
\hline & 0.05 & $5(17.2)$ & $6(20.7)$ & \\
\hline & 0.1 & $12(41.4)$ & $10(34.5)$ & \\
\hline & 0.2 & $5(17.2)$ & $3(10.3)$ & \\
\hline & 0.3 & $0(0.0)$ & $5(17.3)$ & \\
\hline & 0.4 & $2(6.9)$ & $1(3.4)$ & \\
\hline & 0.5 & $1(3.4)$ & $1(3.4)$ & \\
\hline & 0.6 & $2(6.9)$ & $2(7.0$ & \\
\hline & 0.9 & $1(3.4)$ & $1(3.4)$ & \\
\hline \multirow[t]{2}{*}{ Preoperative BCVA in decimal notation, $n(\%)$} & 1 & $28(96.6)$ & $28(96.6)$ & \multirow[t]{2}{*}{0.990} \\
\hline & 1.2 & $1(3.4)$ & $1(3.4)$ & \\
\hline \multicolumn{2}{|l|}{ Age $(y)$, Mean \pm SD } & $26.1 \pm 7.3$ & $26.1 \pm 7.3$ & 0.990 \\
\hline \multicolumn{2}{|l|}{ Mesopic pupil diameter $(\mathrm{mm})$, Mean \pm SD } & $6.3 \pm 0.85$ & $6.3 \pm 0.8$ & 0.990 \\
\hline \multicolumn{2}{|l|}{ CCT, Mean \pm SD } & $547.4 \pm 33.2$ & $547 \pm 33.1$ & 0.980 \\
\hline \multicolumn{2}{|l|}{ SE (D), Median, 25\% quartile (Min, Max) } & $-3.0,-3.9(-6.75,+1.0)$ & $-2.75,-3.9(-6.75,+1.25)$ & 0.970 \\
\hline \multicolumn{2}{|l|}{ K1, Mean \pm SD } & $42.8 \pm 1.4$ & $42.8 \pm 1.4$ & 0.990 \\
\hline \multicolumn{2}{|l|}{ K2, Mean \pm SD } & $43.9 \pm 1.5$ & $44.1 \pm 1.6$ & 0.700 \\
\hline
\end{tabular}

Abbreviations: n, number; \%, percentage; UCVA, uncorrected visual acuity; BCVA, best-corrected visual acuity; y, years; SD, standard deviation; mm, millimeter; CCT, central corneal thickness; SE, refractive error spherical equivalent; D, diopter; min, minimum; max, maximum; K1, keratometry reading in the flattest meridian; K2, keratometry reading in the steepest meridian.

Table 3. Comparison of follow up outcomes after transepithelial photorefractive keratectomy (t-PRK) in eyes fitted with two types of bandage contact lenses

\begin{tabular}{|c|c|c|c|}
\hline Variable & $\begin{array}{l}\text { Comfilcon } A \\
(n=29 \text { eyes })\end{array}$ & $\begin{array}{c}\text { Lotrafilcon B } \\
(n=29 \text { eyes })\end{array}$ & P-value \\
\hline UCVA (Decimal) & Mean $\pm S D$ & Mean $\pm S D$ & \\
\hline Day 1 & $0.43 \pm 0.2$ & $0.44 \pm 0.22$ & 0.610 \\
\hline Day 4 & $0.76 \pm 0.3$ & $0.7 \pm 0.25$ & 0.300 \\
\hline Day 7 & $0.86 \pm 0.16$ & $0.8 \pm 0.2$ & 0.500 \\
\hline Intra group comparison & $P=0.03$ & $P=0.70$ & \\
\hline Pain score & Median, $25 \%$ quartile & Median, $25 \%$ quartile & \\
\hline Day 1 & $4.0,3.0$ & $6.0,3.0$ & $<0.001$ \\
\hline Day 4 & $0.0,0.0$ & $0.0,0.0$ & 0.900 \\
\hline Day 7 & $0.0,0.0$ & $0.0,0.0$ & 0.900 \\
\hline Intra group comparison & $P<0.001$ & $P<0.001$ & \\
\hline Epithelial defect $\left(\mathrm{mm}^{2}\right)$ & Median, $25 \%$ quartile & Median, $25 \%$ quartile & \\
\hline Day $1(n=29)$ & 16,9 & 15,9 & $<0.001$ \\
\hline Day $4(n=29)$ & $0.0,0.0$ & $0.0,0.0$ & 0.900 \\
\hline Day $7(n=11)$ & $0.0,0.0$ & $0.0,0.0$ & 0.900 \\
\hline Intra group comparison & $P<0.001$ & $P<0.001$ & \\
\hline
\end{tabular}

Abbreviations: n, number; UCVA, uncorrected visual acuity; SD, standard deviation; $\mathbf{m m}^{2}$, square millimeters. $P-$ value $<0.05$ is shown in bold. 
A comparison of lotrafilcon B to balafilcon A lenses reported that lotrafilcon B offered more advantages [6]. A recent study conducted by Ozarslan and Ozcan reported faster healing of the corneal epithelium with lotrafilcon B than with balafilcon A after corneal crosslinking [17]. In the present study, the epithelial defects in eyes that received lotrafilcon B lenses were smaller and healed faster (based on daily follow-up of the size of the epithelial deffects by measurement under slit lamp up to complete healing) compared to the eyes that received comfilcon A lenses. These observations differ from those of Grentzelos et al. [18], who reported similar healing times for lotrafilcon B and lotrafilcon A lenses after PRK. Both lenses in Grentzelos et al.s study [18] had high $\mathrm{DK} / \mathrm{t}$ values, which may explain their similar performance after PRK. Careful interpretation of the DK/t values provided by the lens manufacturer is warranted, as a previous study reported a wide variation in these values [19]. However, samfilcon A was superior to lotrafilcon B in accelerating post-PRK epithelial healing and decreasing postoperative pain, according to a study by Yuksel et al. [20]

Stahl and Jalbert concluded in their study that patient discomfort and tear film instability are related to prolonged lens wear, and are not affected by lens type [21]. Another study conducted by Garcia-Mantero et al. concluded that, the types of materials used in lotrafilcon B, samfilcon A, comfilcon A, and filcom V3 silicone hydrogel contact lenses are capable of maintaining the integrity of different ocular surface parameters during and after contact lens wear [19].

The comparison of bandage lenses in contralateral eyes of the same individual following t-PRK may allow reliable conclusions regarding the best choice for bandage lenses after PRK to ensure better comfort and faster healing. Nevertheless, the study had some limitations. The method for assessing epithelial defects in our study was based on slit-lamp bio-microscopy. Other studies used anterior segment optical coherence tomography (OCT) to evaluate epithelial defects [22]. This method allows more accurate documentation and provides ultra-high resolution images. We did not include data concerning contact lens mobility in the postoperative assessment. Centration and mobility of contact lenses fitted on the cornea could provide important information regarding epithelial status [23]. Therefore, further studies are required to detect the best postoperative measures to reduce pain and promote healing, by means of more accurate tools, such as anterior segment OCT, along with observation of contact lens centration and mobility.

\section{CONCLUSIONS}

The present study found that eyes fitted with lotrafilcon B lenses had better healing responses than eyes fitted with comfilcon A lenses in the early postoperative period after t-PRK for low to moderate myopia. Patient perception of discomfort was greater with lotrafilcon B lenses for the first few postoperative days, yet the final outcome was comparable between the two groups.

\section{ETHICAL DECLARATIONS}

Ethics approval: The study protocol was reviewed and approved by the Medical Ethics Committee of Specialised Medical Center Hospital, Riyadh, Saudi Arabia. Written informed consent was obtained from all participants in the study.

Conflict of interests: None.

\section{FUNDING}

\section{None.}

\section{ACKNOWLED GMENT}

We would like to express our gratitude to our patients, professors, and colleagues. This work could not have been accomplished without your support.

\section{REFERENCES}

1. Eliacik M, Erdur SK, Gulkilik G, Ozsutcu M, Karabela Y. Compare the effects of two silicone-hydrogel bandage contact lenses on epithelial healing after photorefractive keratectomy with anterior segment optical coherence tomography. Cont Lens Anterior Eye. 2015;38(3):215-9. doi: 10.1016/j.clae.2015.01.017 pmid: 25707929

2. Sia RK, Ryan DS, Stutzman RD, Psolka M, Mines MJ, Wagner ME, et al. Alcohol versus brush PRK: visual outcomes and adverse effects. Lasers Surg Med. 2012;44(6):475-81. doi: 10.1002/lsm.22036 pmid: 22674627

3. Edwards JD, Bower KS, Sediq DA, Coe CD, Kuzmowych CP, Eaddy JB, et al. Comparison of Visual Outcomes After Epithelial Laser-Assisted Keratomileusis (Epi-Lasik) and Photorefractive Keratectomy (PRK). Investigative Ophthalmology \& Visual Science. 
2009;50(13):553.

4. Ghetemme C, Agapie A, Guechi O, Jeancolas A-L, Lhuillier L, Premy S, et al. Refractive Efficency of Transepithelial Photorefractive Keratectomy (trans-PRK). Investigative Ophthalmology \& Visual Science. 2014;55(13):1527.

5. Ghadhfan F, Al-Rajhi A, Wagoner MD. Laser in situ keratomileusis versus surface ablation: visual outcomes and complications. J Cataract Refract Surg. 2007;33(12):2041-8. doi: 10.1016/j.jcrs.2007.07.026 pmid: 18053901

6. Mohammadpour M, Heidari Z, Hashemi H, Asgari S. Comparison of the Lotrafilcon B and Comfilcon A Silicone Hydrogel Bandage Contact Lens on Postoperative Ocular Discomfort After Photorefractive Keratectomy. Eye Contact Lens. 2018;44 Suppl 2:S273-S6. doi: 10.1097/ICL.0000000000000471 pmid: 29438121

7. Fadlallah A, Fahed D, Khalil K, Dunia I, Menassa J, El Rami H, et al. Transepithelial photorefractive keratectomy: clinical results. J Cataract Refract Surg. 2011;37(10):1852-7. doi: 10.1016/j.jcrs.2011.04.029 pmid: 21840678

8. Yuksel E, Ozulken K, Uzel MM, Taslipinar Uzel AG, Aydogan S. Comparison of Samfilcon A and Lotrafilcon B silicone hydrogel bandage contact lenses in reducing postoperative pain and accelerating re-epithelialization after photorefractive keratectomy. Int Ophthalmol. 2019;39(11):2569-2574. doi: 10.1007/s10792-019-01105-9 pmid: 31065904

9. Bagherian H, Zarei-Ghanavati S, Momeni-Moghaddam H, Wolffsohn JS, Sedaghat MR, Naroo SA, et al. Masked comparison of two silicone hydrogel bandage contact lenses after photorefractive keratectomy. Cont Lens Anterior Eye. 2020;43(3):244-249. doi: 10.1016/j. clae.2020.02.005 pmid: 32098716

10. Plaka A, Grentzelos MA, Astyrakakis NI, Kymionis GD, Pallikaris IG, Plainis S. Efficacy of two silicone-hydrogel contact lenses for bandage use after photorefractive keratectomy. Cont Lens Anterior Eye. 2013;36(5):243-6. doi: 10.1016/j.clae.2013.02.015 pmid: 23522992

11. Morgan PB, Brennan NA, Maldonado-Codina C, Quhill W, Rashid K, Efron N. Central and peripheral oxygen transmissibility thresholds to avoid corneal swelling during open eye soft contact lens wear. J Biomed Mater Res B Appl Biomater. 2010;92(2):361-5. doi: 10.1002/jbm.b.31522 pmid: 19904816

12. Yaylali V, Yildirim C, Tatlipinar S, Demirlenk I, Arik S, Ozden S. Subjective visual experience and pain level during phacoemulsification and intraocular lens implantation under topical anesthesia. Ophthalmologica. 2003;217(6):413-6. doi: 10.1159/000073071 pmid: 14573974

13. Mukerji N, Vajpayee RB, Sharma N. Technique of area measurement of epithelial defects. Cornea. 2003;22(6):549-51. doi: 10.1097/00003226-200308000-00012 pmid: 12883349

14. Bukhari A, Ajlan R, Alsaggaf H. Prevalence of dry eye in the normal population in Jeddah, Saudi Arabia. Orbit. 2009;28(6):392-7. doi: 10.3109/01676830903074095 pmid: 19929667

15. Nilsson SE. Seven-day extended wear and 30-day continuous wear of high oxygen transmissibility soft silicone hydrogel contact lenses: a randomized 1-year study of 504 patients. CLAO J. 2001;27(3):125-36. pmid: 11506437

16. Qiao H, Luensmann D, Heynen M, Drolle E, Subbaraman LN, Scales C, et al. In vitro Evaluation of the Location of Cholesteryl Este Deposits on Monthly Replacement Silicone Hydrogel Contact Lens Materials. Clin Ophthalmol. 2020;14:2821-2828. doi: 10.2147/ opth.s270575 pmid: 33061266

17. Ozarslan Ozcan D, Ozcan SC. Efficacy of two silicone-hydrogel bandage contact lenses after corneal crosslinking. Clin Exp Optom. 2021;104(4):505-509. doi: 10.1080/08164622.2021.1878838 pmid: 33689606

18. Grentzelos MA, Plainis S, Astyrakakis NI, Diakonis VF, Kymionis GD, Kallinikos P, et al. Efficacy of 2 types of silicone hydrogel bandage contact lenses after photorefractive keratectomy. J Cataract Refract Surg. 2009;35(12):2103-8. doi: 10.1016/j.jcrs.2009.07.015 pmid: 19969215

19. Garcia-Montero M, Rico-Del-Viejo L, Llorens-Quintana C, Lorente-Velazquez A, Hernandez-Verdejo JL, Madrid-Costa D. Randomized crossover trial of silicone hydrogel contact lenses. Cont Lens Anterior Eye. 2019;42(5):475-481. doi: 10.1016/j.clae.2018.12.006 pmid: 30587408

20. Yuksel N, Yaman D. Lotrafilcon B with HydraGlyde moisture matrix or Samfilcon A: Contralateral comparison study for comfort. Taiwan J Ophthalmol. 2019;9(2):100-103. doi: 10.4103/tjo.tjo_29_18 pmid: 31198668

21. Stahl U, Jalbert I. Exploring the links between contact lens comfort, osmolarity and lid wiper staining. Cont Lens Anterior Eye. 2018;41(1):110-116. doi: 10.1016/j.clae.2017.09.011 pmid: 28939265

22. Pang CE, M V, Tan DT, Mehta JS. Evaluation of Corneal Epithelial Healing Under Contact Lens with Spectral-Domain Anterior Segment Optical Coherence Tomography (SD-OCT). Open Ophthalmol J. 2011;5:51-4. doi: 10.2174/1874364101105010051 pmid: 21686324

23. Wolffsohn JS, Drew T, Dhallu S, Sheppard A, Hofmann GJ, Prince M. Impact of soft contact lens edge design and midperipheral lens shape on the epithelium and its indentation with lens mobility. Invest Ophthalmol Vis Sci. 2013;54(9):6190-7. doi: 10.1167/iovs.1312425 pmid: 23942975 